\title{
The Borodin-Kostochka conjecture for graphs containing a doubly critical edge
}

\author{
Landon Rabern \\ landon.rabern@gmail.com
}

Submitted: Jul 23, 2007; Accepted: Oct 14, 2007; Published: Oct 24, 2007

Mathematics Subject Classification: 05C15

\begin{abstract}
We prove that if $G$ is a graph containing a doubly-critical edge and satisfying $\chi \geq \Delta \geq 6$, then $G$ contains a $K_{\Delta}$.
\end{abstract}

\section{Introduction}

Way back in 1977, Borodin and Kostochka made the following conjecture (see [1]).

Conjecture. Every graph satisfying $\chi \geq \Delta \geq 9$ contains $a K_{\Delta}$.

Examples exist showing that the $\Delta \geq 9$ condition is necessary (e.g. for the $\Delta=8$ case, take a 5-cycle and expand each vertex to a triangle). In 1999, Reed proved the conjecture for $\Delta \geq 10^{14}$ (see [3]).

Definition 1. Let $G$ be a graph. An edge $a b \in G$ is doubly critical just in case $\chi(G \backslash$ $\{a, b\})=\chi(G)-2$.

We prove the following.

Theorem A. Let $G$ be a graph containing a doubly critical edge. If $G$ satisfies $\chi \geq \Delta \geq 6$, then $G$ contains a $K_{\Delta}$.

To see that this result is tight, consider the following graph. Put $A=\{1,2\}, B=\{3,4,5\}$ and $C=\{6,7,8,9\}$. Let $G$ be the graph having $V(G)=A \cup B \cup C$ with $A$ and $C$ complete, $B$ empty, and the additional edges $13,14,15,23,24,25,64,65,73,75,83,84,93,94$. It is easily checked that $G$ satisfies $\chi=\Delta=5$ and $\omega=4$. Also, $G$ contains a doubly critical edge since removing both vertices 8 and 9 leaves a 3 -chromatic graph. A counterexample with $\chi=\Delta=4$ can be made by removing vertices 1 and 9 from $G$. The theorem holds trivially for $\Delta \leq 3$ since the only triangle-free graph containing a doubly critical edge is $K_{2}$. 
We briefly mention a related conjecture of Lovàsz. He conjectures that the stronger condition that every edge of a connected graph $G$ is doubly critical implies that $G$ is complete (see [1]). Stiebitz has shown that this conjecture holds for graphs with chromatic number at most 5 (see [4]).

\section{The Lonely Path Lemma}

We reproduce the relevant definitions and lemmas from [2].

Definition 2. Let $C=\left\{I_{1}, \ldots, I_{m}\right\}$ be a coloring of a graph $G$. If there exists $j \neq k$ such that $v \in I_{j}, w \in I_{k}$ and $N(v) \cap I_{k}=\{w\}$, then the (directed) edge $(v, w)$ is called $C$-lonely. If the coloring is clear from context we drop the $C$ and just call the edge plain lonely.

The following lemma is clear from the definition of $C$-lonely.

Lemma 2.1. Let $C$ be a coloring of a graph $G$. If both $(v, w)$ and $(w, v)$ are $C$-lonely, then swapping $v$ and $w$ yields a new coloring $C^{\prime}$ with $|C|=\left|C^{\prime}\right|$.

Definition 3. Let $C$ be a coloring of a graph $G$. The $C$-lonely graph of $G$ (denoted $L_{C}(G)$ ) is the directed graph with vertex set $V(G)$ and edge set $\{(v, w) \mid(v, w)$ is $C$-lonely in $G\}$.

Definition 4. Let $C$ be a coloring of a graph $G$. For any vertex $v \in G$, set

$$
L_{C}(v)=\{w \in G \mid(v, w) \text { is C-lonely }\} .
$$

The following is the main lemma from [2]. We reproduce the proof here for completeness.

Lonely Path Lemma. Let $G$ be a graph. If $C$ is an optimal coloring of $G,\{a\},\{b\} \in C$ are distinct singleton color classes and $p_{a}, p_{b}$ are vertex disjoint (directed) paths in $L_{C}(G)$ (starting at $a, b$ respectively) both having at most one vertex in any given color class, then the vertices of $p_{a}$ are completely joined to the vertices of $p_{b}$ in $G$.

Proof. Assume (to reach a contradiction) that the lemma is false. Of all counterexamples, pick an optimal coloring $C$ of $G,\{a\},\{b\} \in C$ distinct singleton color classes and $p_{a}, p_{b}$ vertex disjoint (directed) paths in $L_{C}(G)$ (starting at $a, b$ respectively) both having at most one vertex in any given color class where the sum of the lengths of $p_{a}$ and $p_{b}$ is minimized. Then, by the minimality condition, all but the ends of $p_{a}$ and $p_{b}$ must be joined in $G$. If $p_{a}$ contains more than one vertex ( say $\left.p_{a}=a, a_{2}, a_{3}, \ldots, a_{n}\right)$, then $\left(a, a_{2}\right)$ is lonely since $p_{a}$ is a path in $L_{C}(G)$. But $\{\mathrm{a}\}$ is a singleton color class, so $\left(a_{2}, a\right)$ is also lonely. Hence, by Lemma 2.1, swapping $a$ and $a_{2}$ yields another optimal coloring $C^{\prime}$ of $G$.

To apply the minimality condition, we need to show that $p_{a}^{\prime}=a_{2}, a_{3}, \ldots, a_{n}$ and $p_{b}$ are paths in $L_{C^{\prime}}(G)$. Let $I_{j}, I_{j}^{\prime}$ be the color classes containing $a_{j}$ in $C, C^{\prime}$ respectively. Assume that $p_{a}^{\prime} \notin L_{C^{\prime}}(G)$. Then we have $2 \leq k \leq n-1$ such that $\left|N\left(a_{k}\right) \cap I_{k+1}^{\prime}\right| \neq 1$. Hence $I_{k+1}^{\prime} \neq I_{k+1}$. Since swapping $a$ and $a_{2}$ only changes $\{a\}$ and $I_{2}$, we must have $I_{k+1}=\{a\}$ 
or $I_{k+1}=I_{2}$. In the latter case, $a_{k+1}=a_{2}$ since $p_{a}$ has at most one vertex in each color class. Thus $a_{k+1}=a$ or $a_{k+1}=a_{2}$. If $a_{k+1}=a_{2}$, then $I_{k+1}^{\prime}=\left\{a_{k+1}\right\}$ contradicting the fact that $\left|N\left(a_{k}\right) \cap I_{k+1}^{\prime}\right| \neq 1$. Whence $a_{k+1}=a$. Since $p_{a}$ is a path, it has no repeated internal vertices; hence, $k+1=n$. This is a contradiction since $a_{n}$ is not joined to the end of $p_{b}$ but $a$ is. Whence $p_{a}^{\prime} \in L_{C^{\prime}}(G)$.

Now assume that $p_{b} \notin L_{C^{\prime}}(G)$ (say $\left.p_{b}=b, b_{2}, \ldots, b_{m}\right)$. Let $Q_{j}, Q_{j}^{\prime}$ be the color classes containing $b_{j}$ in $C, C^{\prime}$ respectively. Then we have $2 \leq e \leq m-1$ such that $\left|N\left(b_{e}\right) \cap Q_{e+1}^{\prime}\right| \neq 1$. Hence $Q_{e+1}^{\prime} \neq Q_{e+1}$. Since swapping $a$ and $a_{2}$ only changes $\{a\}$ and $I_{2}$, we must have $Q_{e+1}=\{a\}$ or $Q_{e+1}=I_{2}$. The former is impossible since $p_{a}$ and $p_{b}$ are disjoint. Hence $Q_{e+1}=I_{2}$. Since $e<m, b_{e}$ is adjacent to $a_{2}$. Since $\left|N\left(b_{e}\right) \cap I_{2}\right|=\left|N\left(b_{e}\right) \cap Q_{e+1}\right|=1$, we must have $b_{e+1}=a_{2}$ contradicting the disjointness of $p_{a}$ and $p_{b}$. Whence $p_{b} \in L_{C^{\prime}}(G)$.

Hence $p_{a}^{\prime}$ and $p_{b}$ are vertex disjoint paths in $L_{C^{\prime}}(G)$ with the end of $p_{a}^{\prime}$ not joined to the end of $p_{b}$ and $p_{a}^{\prime}$ shorter than $p_{a}$, contradicting the minimality condition. Hence $p_{a}$ is the single vertex $\{a\}$. Similarly, $p_{b}$ is the single vertex $\{b\}$. Since $p_{a}$ is not joined to $p_{b}$, the color classes $\{a\}$ and $\{b\}$ can be merged, contradicting the fact that $C$ is an optimal coloring.

Lemma 2.2. Let $G$ be a graph and $C=\left\{I_{1}, \ldots, I_{m}\right\}$ an optimal coloring of $G$. Then, for each $1 \leq j \leq m$, there exists $v_{j} \in I_{j}$ such that $N\left(v_{j}\right) \cap I_{k} \neq \emptyset$ for each $k \neq j$.

Proof. Otherwise $C$ would not be optimal.

\section{Proof of The Main Result}

Lemma 3.1. Let $G$ be a graph and $C=\left\{\{a\},\{b\}, I_{3}, \ldots, I_{m}\right\}$ be an optimal coloring of G. Then $N(a) \cap N(b) \cap I_{j} \neq \emptyset$ for $3 \leq j \leq m$.

Proof. Let $3 \leq j \leq m$. By Lemma 2.2, we have $v_{j} \in I_{j}$ such that $a, b \in N\left(v_{j}\right)$.

The following is a simple application of the Lonely Path Lemma to paths of length one.

Lemma 3.2. Let $G$ be a graph and $C=\left\{\{a\},\{b\}, I_{3}, \ldots, I_{m}\right\}$ be an optimal coloring of $G$. Then for any $X \subseteq L_{C}(a) \backslash L_{C}(b)$ and $Y \subseteq L_{C}(b) \backslash L_{C}(a)$ with $|X| \leq 1$ and $|Y| \leq 1$, $X \cup Y \cup L_{C}(a) \cap L_{C}(b)$ induces a clique in $G$.

Lemma 3.3. Let $X$ be a set and $d \geq 3$. If $N_{1}, \ldots, N_{d} \subseteq X$ with $\left|N_{i}\right|=2$ for all $1 \leq i \leq d$, $N_{i} \cap N_{j} \neq \emptyset$ for all $1 \leq i \leq j \leq d$ and $\bigcap_{i=1}^{d} N_{i}=\emptyset$, then $\left|\bigcup_{i=1}^{d} N_{i}\right|=3$.

Proof. Assume (to reach a contradiction) that this is not the case and let $N_{1}, \ldots, N_{d}$ be a counterexample with $d$ minimal. Plainly, $d \geq 4$. By the minimality of $d$, the $N_{i}$ are distinct. If $\left\{x_{1}, y_{1}\right\}=N_{1} \nsubseteq \bigcup_{i=2}^{d} N_{i}$, then, without loss of generality, $x_{1} \notin \bigcup_{i=2}^{d} N_{i}$. Hence 
$x_{1} \notin N_{i}$ for $2 \leq i \leq d$. But $N_{1}$ has non-trivial intersection with each of $N_{2}, \ldots, N_{d}$, so we must have $x_{2} \in N_{i}$ for $2 \leq i \leq d$. Thus $x_{2} \in \bigcap_{i=1}^{d} N_{i}$, giving a contradiction. Whence $N_{1} \subseteq \bigcup_{i=2}^{d} N_{i}$. By the minimality of $d$, the lemma holds for $N_{2}, \ldots, N_{d}$. If $\bigcap_{i=2}^{d} N_{i}=\emptyset$, then $\left|\bigcup_{i=2}^{d} N_{i}\right|=3$. But $N_{1} \subseteq \bigcup_{i=2}^{d} N_{i}$ giving $\left|\bigcup_{i=1}^{d} N_{i}\right|=3$, a contradiction. Hence we have $z_{1} \in \bigcap_{i=2}^{d} N_{i}$. Similarly, we have $z_{2} \in N_{1} \cap \bigcap_{i=3}^{d} N_{i}$ and $z_{3} \in N_{1} \cap N_{2} \cap \bigcap_{i=4}^{d} N_{i}$. Since $\left\{z_{1}, z_{2}, z_{3}\right\} \subseteq N_{4}$ and $\left|N_{4}\right|=2$, two of the $z$ 's coincide. Without loss of generality assume $z_{1}=z_{2}$. Then $z_{1} \in \bigcap_{i=1}^{d} N_{i}$ giving a final contradiction.

Proof of Theorem A. Assume (to reach a contradiction) that $G$ satisfies $\chi \geq \Delta \geq 6$ and does not contain a $K_{\Delta}$. Without loss of generality, we may assume that $G$ is connected. By Brooks' theorem we must have $\chi(G)=\Delta(G)$. Set $m=\chi(G)$ and let $C=\left\{\{a\},\{b\}, I_{3}, \ldots, I_{m}\right\}$ be an optimal coloring of $G$. By Lemma 2.2, $a$ is adjacent to at least one vertex in each element of $C \backslash\{a\}$. Hence $m-1 \leq d(a) \leq \Delta(G)=m$ and thus $m-2 \leq\left|L_{C}(a)\right| \leq m-1$. Similarly, $m-2 \leq\left|L_{C}(b)\right| \leq m-1$. If $\left|L_{C}(a) \cup L_{C}(b)\right|=m$, then a straightforward application of Lemma 3.2 produces a $K_{m}$ in $G$. Thus we have $\left|L_{C}(a) \cup L_{C}(b)\right| \leq m-1$. Since $b \in L_{C}(a)$ and $a \in L_{C}(b)$, we must have $\left|L_{C}(a) \cup L_{C}(b)\right|=m-1$. Let $K$ be the unique color class that $L_{C}(a) \cup L_{C}(b)$ does not hit. Then $|N(a) \cap K|=2$ and $|N(b) \cap K|=2$.

Given $x \in L_{C}(a) \cup L_{C}(b) \backslash\{a, b\}$, both $(a, x)$ and $(x, a)$ are $C$-lonely. Hence, by Lemma 2.1, we may swap $x$ and $a$ to yield a new optimal coloring $C^{\prime}$. By an argument similar to above we conclude that $\left|L_{C^{\prime}}(x) \cup L_{C^{\prime}}(b)\right|=m-1$. Since $K$ is still a color class in $C^{\prime}$ and $b$ hits two elements of $K$, we conclude that $|N(x) \cap K|=2$.

Let $S=L_{C}(a) \cup L_{C}(b)$. By Lemma 3.2, $S$ induces a clique of order $m-1$. For $y \in S$, put $P_{y}=N(y) \cap K$. From the above we know that for each $y \in S$ we have $\left|P_{y}\right|=2$. If there exists $z \in \bigcap_{y \in S} P_{y}$, then $S \cup\{z\}$ induces a $K_{m}$, giving a contradiction. Hence $\bigcap_{y \in S} P_{y}=\emptyset$. Given distinct $y_{1}, y_{2} \in S$, we may swap $y_{1}$ with $a$ and $y_{2}$ with $b$ and apply Lemma 3.1, to conclude that $P_{y_{1}} \cap P_{y_{2}}=N\left(y_{1}\right) \cap N\left(y_{2}\right) \cap K \neq \emptyset$. Now applying Lemma 3.3 with $X=K$ and $\left\{N_{1}, \ldots, N_{d}\right\}=\left\{P_{y} \mid y \in S\right\}$ gives $\left|\bigcup_{y \in S} P_{y}\right|=3$.

Put $T=\bigcup_{y \in S} P_{y}$. Let $A=G \backslash K \backslash S$. Since $S$ induces a clique of order $m-1$ and $\left|P_{y}\right|=2$ for all $y \in S$, there are $m$ edges from each $y \in S$ to $S \cup K$ and hence there are no edges 
between $A$ and $S$. Plainly, $A$ is $m-3$ colorable. Put $H=G[V(A) \cup K]$. We show that $H$ has an $m-1$ coloring in which each element of $T$ receives a different color. There are at most $3 \Delta(G)$ edges from $T$ to $G \backslash K$ and exactly $2|S|=2(\Delta(G)-1)$ edges from $T$ to $S$. Hence there are at most $\Delta(G)+2$ edges from $T$ to $H$. Let $\left\{c_{1}, \ldots, c_{m-3}\right\}$ be a coloring of $A$. If each element of $T$ hit all the $c_{i}$, then the number of edges from $T$ to $S$ would be at least $3(m-3)=3(\Delta(G)-3)$. Hence we would have $3(\Delta(G)-3) \leq \Delta(G)+2$ and thus $\Delta(G) \leq \frac{11}{2}$. Whence, since $\Delta(G) \geq 6$, we have $z \in T$ and $1 \leq i \leq m-3$ such that $z$ misses color $c_{i}$. Let $c_{m-2}$ and $c_{m-1}$ be two new colors. Coloring $z$ with $c_{i}$, the other two elements of $T$ with $c_{m-2}$ and $c_{m-1}$ and the rest of $K$ with $c_{m-2}$ gives an $m-1$ coloring $D$ of $H$ in which each element of $T$ receives a different color.

We now extend $D$ to $S$ using Hall's theorem. Note from above that $|S|=m-1$. For each $y \in S$, let $l_{y}$ be the elements of $\left\{c_{1}, \ldots, c_{m-1}\right\}$ not appearing on an element of $P_{y}$. Then for $y \in S$ we have $\left|l_{y}\right|=m-3$ since $\left|P_{y}\right|=2$. Hence all we need to check is that the union of any $m-2$ of lists has at least $m-2$ elements and that the union of all of the lists has $m-1$ elements. If the former were false, then since $T$ receives three distinct colors under $D$, we would have $y_{1}, \ldots, y_{m-2} \in S$ with $P_{y_{i}}=P_{y_{j}}$ for all $1 \leq i<j \leq m-2$. But the remaining element of $S$ must be adjacent to at least one of the elements of $P_{y_{1}}$ giving a $K_{m}$ in $G$. If the union of all the lists had fewer than $m-1$ elements then we would have $P_{w}=P_{y}$ for all $w, y \in S$ giving a $K_{m}$ once again. Hence Hall's theorem gives distinct $c_{y} \in l_{y}$ for $y \in S$. Since there are no edges between $A$ and $S$, coloring $y$ with $c_{y}$ extends $D$ to an $m-1$ coloring of $G$. This final contradiction completes the proof.

\section{Acknowledgments}

Thanks to the anonymous referee for pointing out that the original $\Delta \geq 9$ condition in Theorem A could be weakened and for suggesting the use of Hall's theorem to finish off the proof.

\section{References}

[1] Tommy R. Jenson and Bjarne Toft. Graph Coloring Problems Wiley., 1995.

[2] Landon Rabern. Coloring and the Lonely Graph. arXiv:070\%.1069, 2007.

[3] Bruce Reed. A strengthening of Brooks' Theorem. J. Combinatorial Th. (B), 76, 1999, $136-149$.

[4] Michael Stiebitz. $K_{5}$ is the only double-critical 5-chromatic graph. Discrete Math., 64, 1987, 91-93. 